\title{
Xenopus Sprouty2 inhibits FGF-mediated gastrulation movements but does not affect mesoderm induction and patterning
}

\author{
Stephen L. Nutt, ${ }^{1,3}$ Kevin S. Dingwell, ${ }^{2,3}$ Christine E. Holt, ${ }^{2}$ and Enrique Amaya ${ }^{1,4}$ \\ ${ }^{1}$ Wellcome/CRC Institute, Cambridge CB2 1QR, UK and Department of Zoology, University of Cambridge CB2 3EJ, UK; \\ ${ }^{2}$ Department of Anatomy, University of Cambridge, Cambridge CB2 3DY, UK
}

Signal transduction through the FGF receptor is essential for the specification of the vertebrate body plan. Blocking the FGF pathway in early Xenopus embryos inhibits mesoderm induction and results in truncation of the anterior-posterior axis. The Drosophila gene sprouty encodes an antagonist of FGF signaling, which is transcriptionally induced by the pathway, but whose molecular functions are poorly characterized. We have cloned Xenopus sprouty2 and show that it is expressed in a similar pattern to known FGFs and is dependent on the FGF/Ras/MAPK pathway for its expression. Overexpression of Xsprouty2 in both embryos and explant assays results in the inhibition of the cell movements of convergent extension. Although blocking FGF/Ras/MAPK signaling leads to an inhibition of mesodermal gene expression, these markers are unaffected by Xsprouty2, indicating that mesoderm induction and patterning occurs normally in these embryos. Finally, using Xenopus oocytes we show that Xsprouty2 is an intracellular antagonist of FGF-dependent calcium signaling. These results provide evidence for at least two distinct FGF-dependent signal transduction pathways: a Sprouty-insensitive Ras/MAPK pathway required for the transcription of most mesodermal genes, and a Sprouty-sensitive pathway required for coordination of cellular morphogenesis.

[Key Words: Sprouty; FGF signaling; gastrulation; convergent extension]

Received October 9, 2000; revised version accepted February 19, 2001.

Growth factor-induced signal transduction is one of the principle mechanisms by which the vertebrate embryo coordinates cell growth, differentiation, and morphogenesis. Studies in the amphibian Xenopus laevis have implicated a number of signaling pathways in the inductive events leading to the formation and patterning of the mesoderm. Members of the fibroblast growth factor (FGF) family of secreted polypeptides have the ability to induce mesoderm in naïve ectodermal tissue, a capacity shared with TGF- $\beta$-type proteins (Kimelman and Griffin 2000). Moreover, the expression of a dominant negative form of the FGF receptor (dnFGFR) at the time of mesoderm induction completely blocks this process in vivo (Amaya et al. 1991; 1993). FGF receptor signaling is also thought to be involved in the subsequent maintenance of the mesodermal tissue as the expression of dnFGFR after the initial induction again results in the loss of mesoder-

\footnotetext{
${ }^{3}$ These authors contributed equally to this work.

${ }^{4}$ Corresponding author.

E-MAIL ea3@mole.bio.cam.ac.uk; FAX +44 (01223) 334-089.

Article and publication are at www.genesdev.org/cgi/doi/10.1101/gad. 191301.
}

mal markers (Kroll and Amaya 1996). This maintenance function is a result of an autocrine loop, which involves the activation of Xbrachrury (Xbra) expression by FGF signaling, Xbra in turn induces eFGF transcription, which reinforces the mesodermal fate of the induced tissue (Isaacs et al. 1994; Schulte-Merker and Smith 1995).

Once mesoderm is formed, gastrulation can proceed through the orchestrated movement of the three germ layers, to produce the anterior-posterior (A-P) axis of the embryo. These cell movements involve both involution and convergent extension. Convergent extension is characterized by the polarization of the mesodermal cells and their mediolateral intercalation to produce a pronounced elongation of the A-P axis (Keller 1991; Keller et al. 1992). The mechanisms by which convergent extension and gastrulation as a whole are coordinated remain poorly understood, although recent evidence has implicated signaling through a noncanonical Wnt pathway (Djiane et al. 2000; Heisenberg et al. 2000; Tada and Smith 2000; Wallingford et al. 2000). FGFs constitute a second signal transduction pathway, which has also been implicated in the processes of gastrulation morphogenesis (for review, see Rossant et al. 1997). Xenopus em- 
bryos overexpressing the dnFGFR fail to undergo gastrulation; however, the absence of mesoderm in these embryos has made it impossible to determine whether FGF signaling has a direct role in morphogenesis, or if the observed gastrulation defects are secondary to the failure of mesoderm formation.

FGFs constitute a family of peptide growth factors, which with binding to their receptors (FGFR), induce receptor dimerization and autophosphorylation of a number of intracellular tyrosine residues (Mohammadi et al. 1996). These phosphorylated tyrosines then serve as docking sites for Src homology 2 (SH2) domain-containing proteins such as phospholipase C- $\gamma($ PLC- $\gamma$ ), which binds specifically to phosphotyrosine 766 (Y766) and stimulates the phosphatidylinositol hydrolysis and the mobilization of $\mathrm{Ca}^{2+}$ in the cell (Mohammadi et al. 1992; Peters et al. 1992). Another consequence of receptor autophosphorylation is to activate the GTPase Ras, setting off a cascade of kinases including Raf, MEK, and finally MAPK, which ultimately results in processes such as the induction of gene expression (Fambrough et al. 1999). FGF-mediated mesoderm induction in Xenopus requires an intact Ras/MAPK pathway, as dominantnegative versions of Ras (Whitman and Melton 1992), Raf (MacNicol et al. 1993), the src-like kinase laloo (Weinstein et al. 1998), the p85 subunit of the phosphatidylinositol 3'-kinase (PI3K) (Carballada et al. 2001), the adapter molecules Nck and Grb2 (Gupta and Mayer 1998), or the tyrosine phosphatase Shp-2 (SH-PTP2; Tang et al. 1995) block Xbra expression and mesoderm formation. In contrast, the binding of PLC- $\gamma$ to Y766 is not essential for this process (Muslin et al. 1994).

Receptor tyrosine kinase (RTK) signaling is regulated at a number of levels including ligand availability, phosphatase activity, and Ras inhibitory proteins such as RasGAP. Another inhibitor of FGF signaling, termed Sprouty (Spry), has been identified in Drosophila (Hacohen et al. 1998). Spry plays a role in the development of the apical branching pattern of Drosophila airways, a process known to require FGF. spry mutations lead to the growth of multiple fine branches from the stalks of the primary branches (Hacohen et al. 1998), a phenotype similar to that observed when FGF signaling is hyperactive, suggesting that Spry inhibits FGFR activity. Moreover, the FGF pathway induces the expression of spry. Because Spry acts noncell autonomously in this system, it was proposed that Spry is a secreted antagonist to FGF, limiting the range of FGF responsiveness in the developing tracheal system (Hacohen et al. 1998). Subsequent genetic screens have revealed a broader function of Sprouty in inhibiting a variety of RTKs such as the Drosophila epidermal growth factor receptor (EGFr) and Torso (Casci et al. 1999). These predominantly genetic studies also suggested that instead of acting extracellularly, Spry acts intracellularly to inhibit the Ras/MAPK pathway (Casci et al. 1999; Reich et al. 1999). Several proposals as to the precise position at which Spry impinges on the FGF pathway have been suggested ranging from a receptor proximal mode of regulation through interactions with Ras, to regulating Raf or molecules fur- ther downstream (Casci et al. 1999; Kramer et al. 1999; Reich et al. 1999). A number of vertebrate Sprouty homologs have been identified; however, little molecular evidence as to their mode of action has yet been presented (de Maximy et al. 1999; Minowada et al. 1999; Tefft et al. 1999; Chambers et al. 2000).

Understanding the role of FGF signaling in vertebrate development requires the identification of its downstream targets. Because Spry is both a target of FGF activity in Drosophila and an inhibitor of the process, we were interested in investigating the functions of Sprouty proteins in the FGF-mediated processes of mesoderm induction and gastrulation in the Xenopus embryo. To this end, we have cloned a Xenopus sprouty homolog Xsprouty2, which is expressed in a similar pattern to that of known FGF signaling molecules. We present biochemical evidence that Xsprouty2 is a component of the FGF signaling cascade, inhibiting the ability of the FGFR to stimulate the release of calcium from intracellular stores. In contrast to observations in Drosophila, Xsprouty2 does not block the Ras/MAPK pathway, and as a result does not prevent mesoderm induction and patterning in developing Xenopus gastrulae. Instead, Xsprouty2 acts to prevent convergent extension movements of the embryo during gastrulation. Therefore, Xsprouty2 provides the first molecular dissection of the distinct roles for FGF signaling in Xenopus mesoderm induction and morphogenesis.

\section{Results}

Isolation of Xenopus sprouty2 genes

To isolate the Xenopus laevis homologs of the human SPROUTY genes, we screened a head library (HemmatiBrivanlou et al. 1991) with the carboxy-terminal region of hSPRY2 cDNA (Hacohen et al. 1998). Two types of clones were isolated, which were highly related to the chick $(75-76 \%)$, mouse $(73-75 \%)$, and human $(74-76 \%)$ Sprouty2 proteins (Fig. 1). The clones designated Xsprouty2

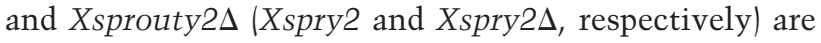
$93 \%$ related at the amino acid level and probably represent paralogs identified as a result of Xenopus laevis tetraploidy. Xspry2 and Xspry2s are 314 and 307 amino acids, respectively, which differ by 20 amino acid substitutions, mostly in the less conserved amino terminal region along with a 7-amino-acid deletion in Xspry2s as compared to all other vertebrate homologs identified (Fig. 1).

\section{Xsprouty2 expression during development}

Analysis of the temporal expression of Xspry2 and Xspry2 $\Delta$ by RT-PCR indicated that both paralogs were broadly expressed throughout development. Xspry2 was induced at the onset of zygotic transcription at the midblastula transition. Thereafter, expression levels remained relatively constant until at least stage 42 . Xspry2s, on the other hand, was expressed at levels approximately 
Nutt et al.

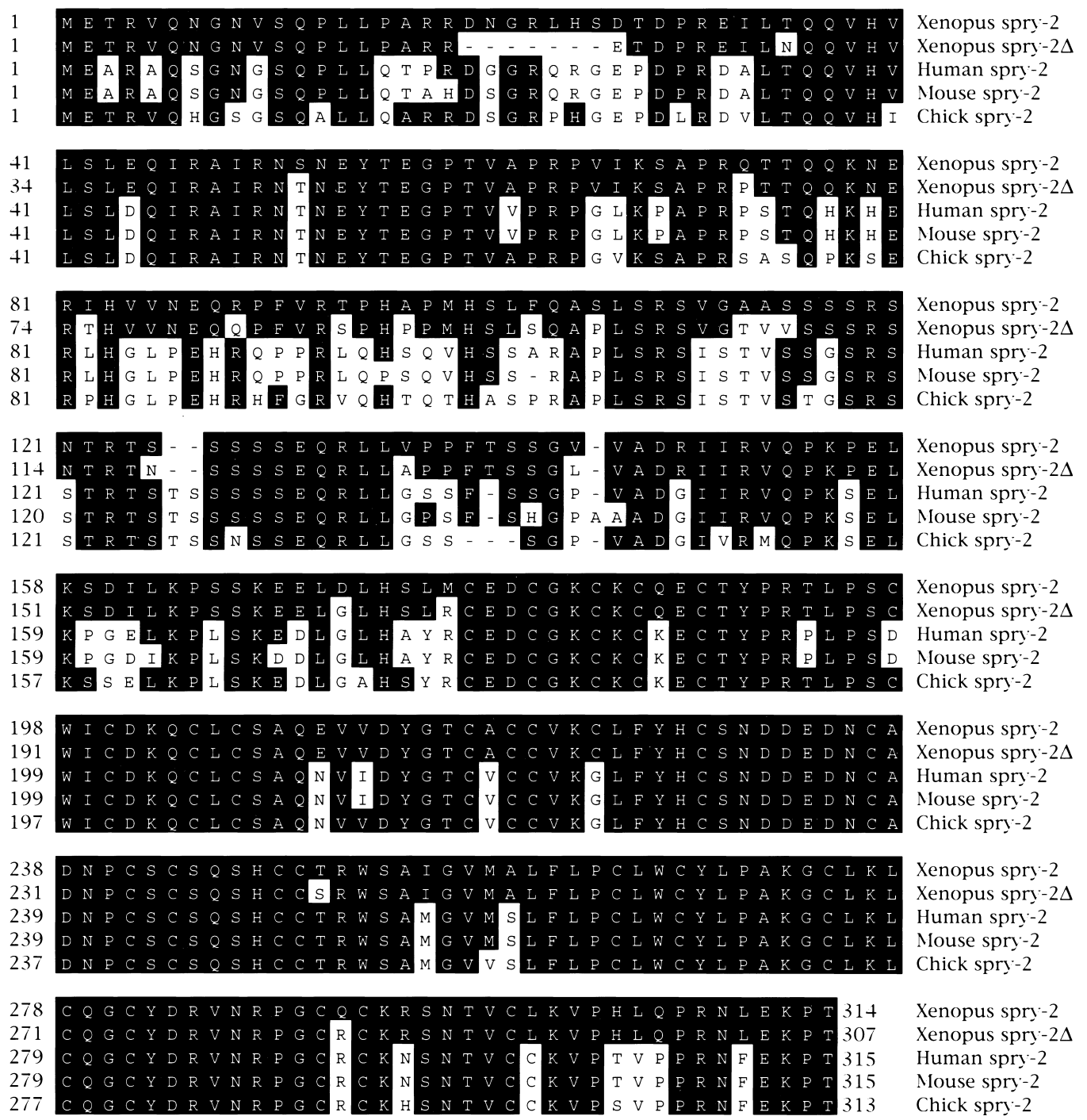

Figure 1. Amino acid sequence comparison of vertebrate Sprouty proteins. The deduced amino acid sequences of Xenopus Xspry2 and Xspry2 $\Delta$ were aligned with the human, mouse, and chick Spry2 proteins (Hacohen et al. 1998; Minowada et al. 1999). Dashes represent gaps inserted to maximize alignment. Amino acids conserved with Xspry2 are indicated by bold overlay.

threefold lower than Xspry2 throughout early development (data not shown).

To determine the spatial pattern of expression of Xspry2, we have analyzed the Xspry2 and Xspry2s (termed Xspry2 when examined collectively) mRNA levels by in situ hybridization. Xspry2 mRNA was first detected before gastrulation in the dorsal marginal zone (data not shown). At the onset of gastrulation Xspry2 expression expanded dorsolaterally and subsequently, was found throughout the marginal zone (Fig. 2A), in a pattern similar to XFGF8 (Christen and Slack 1997), XeFGF (Isaacs et al. 1994), and XFGF9 (Song and Slack 1996). From the mid-neurula stage, Xspry2 was expressed predominantly in the head region (Fig. 2B). By stage 28, Xspry2 mRNA was localized to the developing otic vesicle, the midbrain/ hindbrain isthmus, forebrain, hatching gland, branchial arches, and tail bud, and expression pattern reminiscent of XFGF8 (Fig. 2B). Interestingly, Xspry2 showed a broader staining than that of FGF8, consistent with a role of secreted FGF8 in regulating Xspry2 expression (cf. Fig. $2 \mathrm{~B}, \mathrm{C})$. In summary, Xspry2 is expressed in a dynamic pattern throughout gastrulation and neural development in a manner resembling that of XFGF8.

\section{FGF receptor-mediated signaling is required for Xsprouty2 expression during gastrulation}

The initial characterization of Drosophila spry indicated that the gene itself is a target of RTK signaling (Hacohen et al. 1998). To test whether the Xspry2 genes we have isolated require FGF signaling for their expression, we disrupted FGF signaling by injecting the dnFGFR mRNA into one blastomere of two- or four-cell embryos and assayed for Xspry2 mRNA during gastrulation. In the absence of FGF signaling, there was a total loss of Xspry2 expression (Fig. 3), whereas the control HAV $\varnothing$ mRNA (a 


\section{A Xsprouty2}
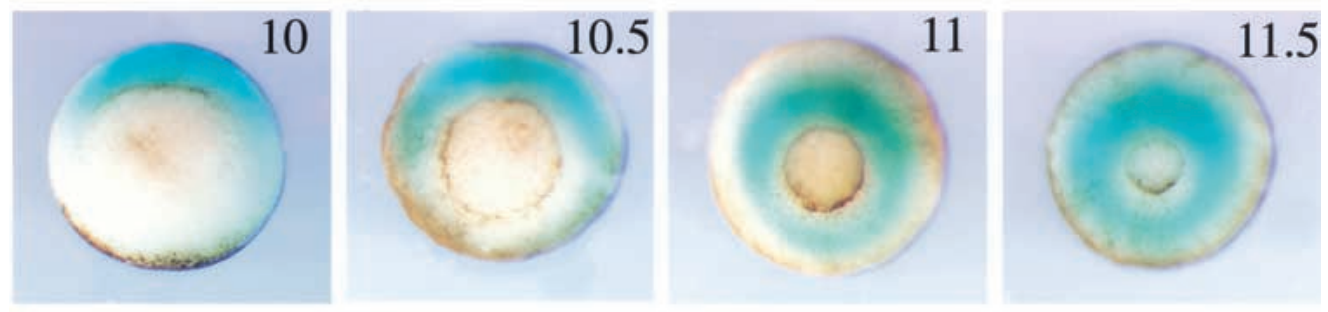

\section{B Xsprouty2}
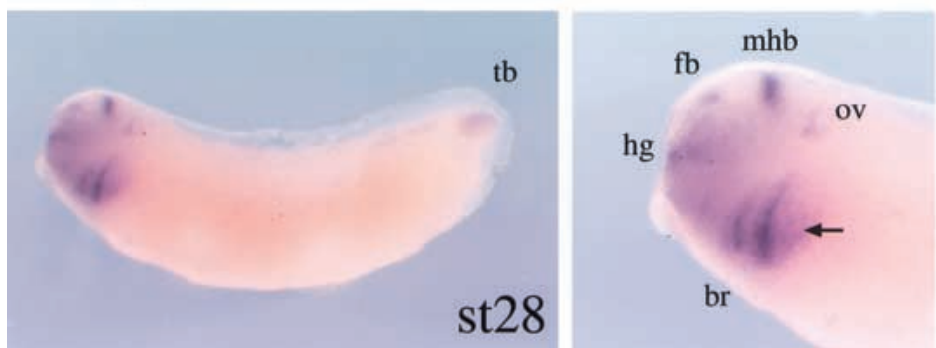

C XFGF8

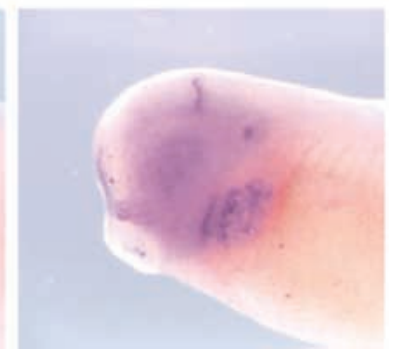

Figure 2. Xsprouty2 is expressed dynamically during early development. Whole mount in situ hybridization of Xspry2 and XFGF8. (A) Vegetal view of gastrula stage embryos (stages 10-11.5). Xspry2 expression is induced in the dorsal marginal zone. Expression expands ventrally around the marginal zone during mid to late gastrula stages. (B) Xspry2 expression in a stage 28 albino embryo and close-up of the head region. Xspry2 can be detected primarily in anterior neural structures, such as the mid-brain/hind-brain isthmus $(\mathrm{mhb})$, otic vesicle (ov), forebrain (fb), and hatching gland (hg). Lateral expression is seen in the branchial arches (br) and the tail bud (tb). (C) Close-up of head region of a stage 28 embryo stained for XFGF8. XFGF8 has a similar expression profile to Xspry2, although with more sharply defined boundaries. Black arrow indicates expression in the third branchial arch, which is absent from the Xspry2 expression profile.

nonfunctional FGF receptor construct) had no effect on Xspry2 mRNA levels. To test whether this induction was through the Ras/MAPK pathway we expressed a dominant negative Ras protein (dnRas) in a similar manner during gastrulation. Expression of dnRas also completely abolished Xspry2 expression indicating that like Xbra, Xspry2 is a target of Ras/MAPK signaling (Fig. 3). In contrast, we have been unable to demonstrate a robust induction of $X$ spry2 in animal caps by eFGF or an ectopic induction of Xpry2 in embryos after eFGF or FGF8 misexpression (data not shown), suggesting that FGF signaling may be necessary but not sufficient for Xspry2 activation. Alternatively, there may be signals outside the marginal zone of the embryo that attenuate the ability of FGF to induce $X$ spry2.

Xsprouty2 misexpression leads to a truncated phenotype in embryos

Functional studies in Drosophila suggest that Spry is an inhibitor of RTK signaling through the MAPK pathway. To test whether Xspry2 acts in a similar manner in vertebrates, we overexpressed Xspry2 in the early Xenopus embryo, a developmental system known to require RTK signals emanating from the FGFR (Amaya et al. 1991, 1993). Injection of Xspry2 or Xspry2s mRNA into the
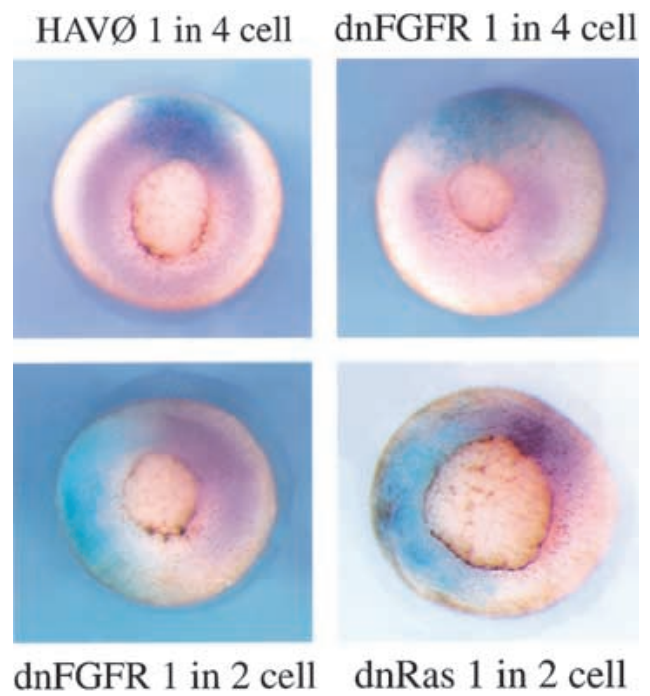

Figure 3. Xsprouty2 expression requires FGF signaling. Vegetal view of in situ hybridization to Xspry2 (purple) at the midto-late gastrula stages (stages 11-11.5). Embryos were injected into one blastomere at the two- or four-cell stages with $1 \mathrm{ng}$ of HAV $\varnothing$, dnRas, or dnFGFR together with 500 pg of Lacz mRNA, and stained for $\beta$-galactosidase activity (blue) as a lineage tracer. 
dorsal marginal zone of a single blastomere at the twocell stage led to a highly reproducible shortening of the A-P axis at stage $35 / 36$, with a low incidence of embryos exhibiting a complete failure of blastopore closure (5$10 \%$; Fig. 4A,B). In contrast, injection of dnFGFR mRNA under the same conditions resulted in a much more severe defect characterized by failure of blastopore closure in all embryos ( $n=16$; Amaya et al. 1991, 1993). Injection of $1 \mathrm{ng}$ of Xspry2 or Xspry2s mRNA into the two dorsal blastomeres at the four-cell stage increased the incidence of incomplete blastopore closure (10-30\%) at stage $35 / 36$ (data not shown), indicating that the severity of the phenotype observed depended on the exact injection regimen used. Therefore, the shortening of the A-P axis produced after Xspry2 overexpression were consistent with a role for Xspry2 in inhibiting FGFR-dependent signaling, but distinct from the complete failure of gastrulation observed in dnFGFR overexpressing embryos.

\section{Xsprouty2 inhibits convergent extension}

To test whether the observed posterior truncations were a result of a failure of Xspry2 overexpressing embryos to undergo convergent extension movements, we conducted lineage tracing experiments. Embryos were injected at the four-cell stage with Xspry2/GFP or the control HAV $\varnothing / G F P$ mRNAs into one dorsal blastomere and rhodamine dextran (RLDX) into the adjacent dorsal blastomere. At stage 40, the majority of the control HAV $\varnothing$ injected embryos showed equal extension of the GFP and rhodamine fluoresence along the entire A-P axis $193 \%$; $n=102$ ), whereas only a minority of the Xspry2-injected embryos showed normal elongation of the GFP-expressing tissue $(17 \% ; n=68)$. The remainder of the Xspry2injected embryos displayed a variety of phenotypes including the restriction of the GFP to the head region resulting in a kink of the axis toward the Xspry2 injected side $(34 \%)$, and a general truncation of both sides of the embryo $(49 \%)$. Both phenotypes are compatible with a failure of the injected cells to extend along the A-P axis (see Fig. 4C for representative embryos).

To further investigate the function of Xspry2 proteins in gastrulation we have used the capacity of animal cap explants to undergo FGFR-dependent convergent extension movements. Animal caps excised from stage 8 blastulae underwent mesoderm induction and convergent extension at a high frequency after treatment with 10 $\mathrm{ng} / \mathrm{ml}$ activin (Fig. 5). This characteristic morphological change was unaffected by injection of the control HAV $\varnothing$ construct and the lineage tracer GFP. However, overexpression of dnFGFR completely inhibited these movements and blocked the ability of the caps to induce and maintain mesoderm (Fig. 5; Cornell and Kimelman 1994). In agreement with our findings in the intact embryo, the injection of Xspry2 or Xspry2s mRNA also inhibited convergent extension movements (Fig. 5A,D). Surprisingly, unlike dnFGFR-injected explants, those expressing Xspry2 induced mesoderm, as shown by the transcription of the mesodermal marker Xbra (Fig. 5C). Similar inhibition of convergent extension but not $\mathrm{Xbra}$ expression was also observed in Xspry2-injected explants treated with FGF2 (data not shown). Interestingly, Xspry2-expressing caps, unlike those injected with dnFGFR, showed some weak morphogenetic movements

A

Figure 4. Overexpression of Xsprouty2 leads to a shortened A-P axis. (A) Embryos were injected once into the dorsal marginal zone at the twocell stage, with $1 \mathrm{ng}$ of the indicated mRNA plus $100 \mathrm{pg}$ of GAP43-GFP mRNA and analyzed at stage 25. (B) Quantitation of the truncated phenotype of injected embryos. Embryos were measured and grouped into three 5 -mm categories. Whereas virtually all noninjected and HAV $\varnothing$-injected embryos were more than $4.3 \mathrm{~mm}$, the majority of Xspry2 and Xspry $2 \Delta$ overexpressing embryos were less than $3.8 \mathrm{~mm}$. $(n)$ the number of embryos examined for each treatment. $(C)$ Representative embryos injected with RLDX and Xspry2/GAP43-GFP or HAV $\varnothing / G A P 43-G F P$ into the dorsal blastomeres at the four-cell stage and allowed to development until stage 40 . The majority of HAV $\varnothing / \mathrm{GFP}$ embryos showed a full extension of GFP-labeled cells dorsally along the A-P axis, whereas Xspry2/GFP-injected embryos showed a kinking of the body toward the injected side $(t o p)$ or a truncation and generalized disorganization of the GFP-positive cells (bottom).

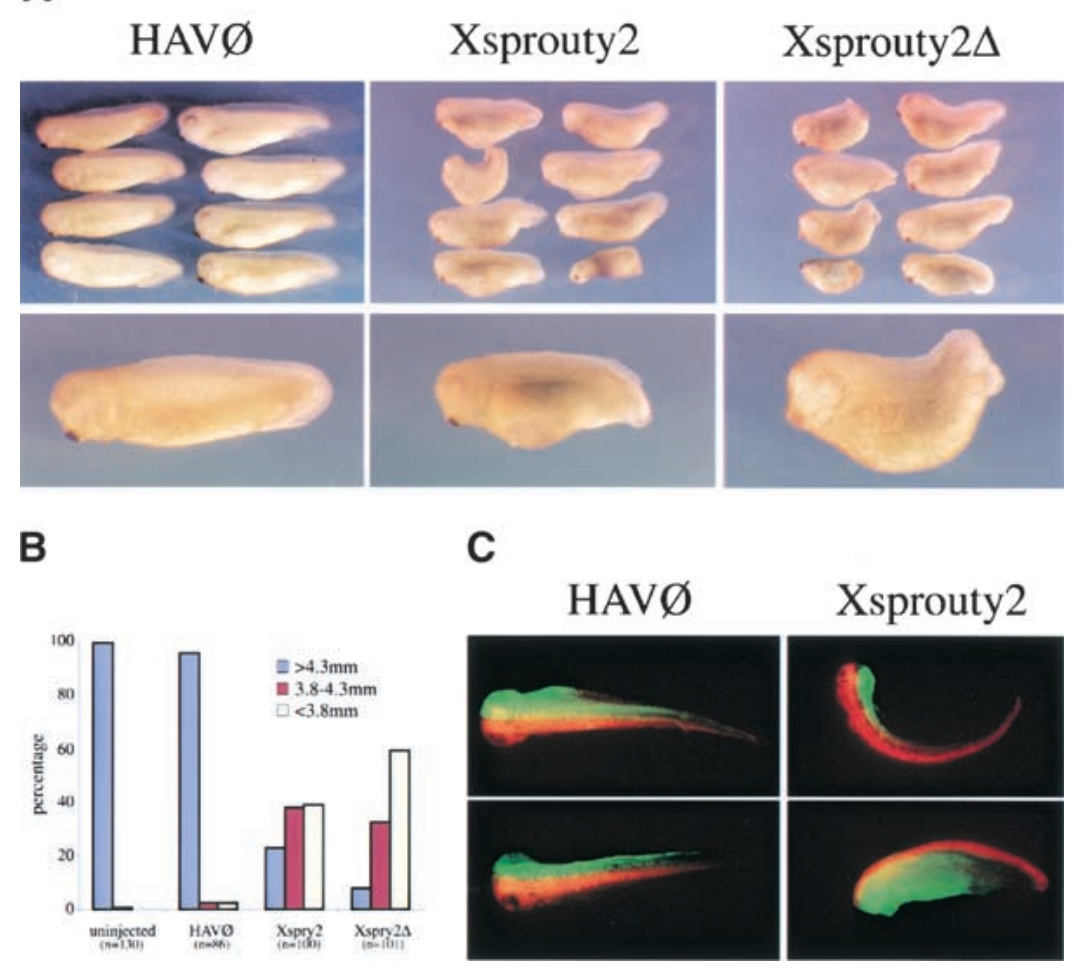


A
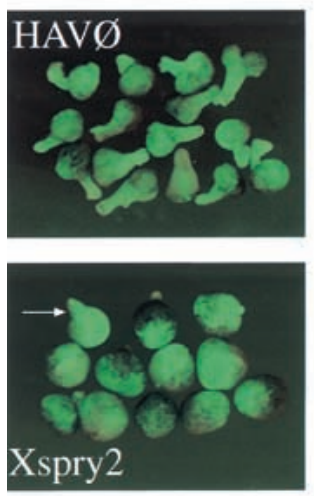

B

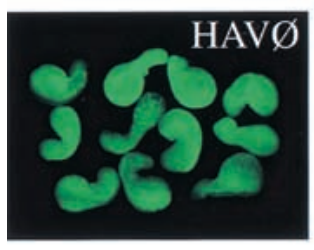

C

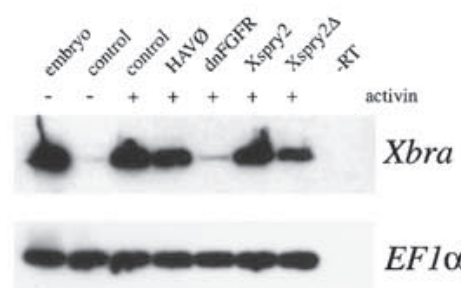

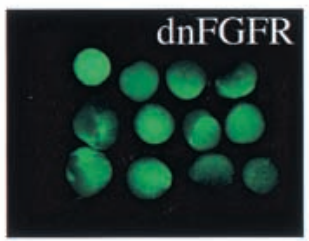
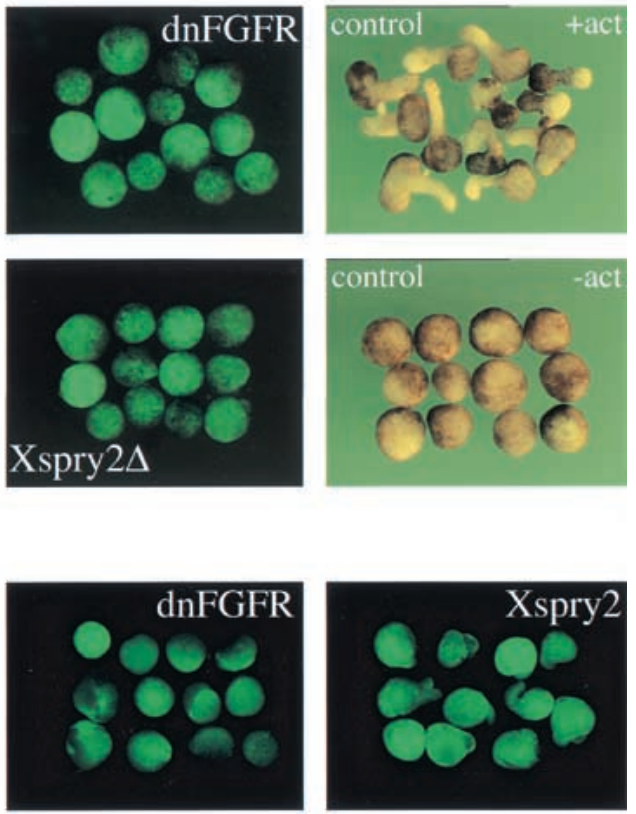

D

\begin{tabular}{lrrrr}
\hline & \multicolumn{3}{c}{ \% elongation } \\
\cline { 2 - 5 } Treatment & + & + & $n$ \\
\hline animal caps & & & & \\
control - activin & 100 & - & - & 49 \\
control + activin & - & 4 & 96 & 85 \\
HAV + activin & 2 & 34 & 64 & 103 \\
dnFGFR + activin & 100 & - & - & 51 \\
Xspry2 + activin & 79 & 20 & 1 & 111 \\
Xspry2 + activin & 98 & 2 & - & 65 \\
Xspry2-N +activin & 7 & 9 & 84 & 68 \\
Xspry2-C +activin & 7 & 16 & 77 & 75 \\
dspry +activin & 83 & 17 & - & 59 \\
dorsal marginal zone explants & 12 & 29 & 59 & 34 \\
HAVØ & 70 & 21 & 9 & 33 \\
dnFGFR & 55 & 35 & 10 & 37 \\
Xspry2 & \multicolumn{5}{c}{}
\end{tabular}

Figure 5. Xsprouty2 inhibits convergent extension but not mesoderm induction. (A) Embryos were injected as indicated with $500 \mathrm{pg}$ of mRNA along with $100 \mathrm{pg}$ of GAP43-GFP mRNA into the animal pole of both blastomeres at the two-cell stage. Animal caps were excised at stage 8 and cultured until stage 18 in the presence of $10 \mathrm{ng} / \mathrm{ml}$ activin, before being examined for GFP fluorescence and elongation. Control noninjected caps were incubated with (+act) or without (-act) activin. All images, except for the control animal caps were taken under fluorescence. (B) Injections were carried out as in $A$, with the exception that they were targeted to the DMZ. DMZ explants were excised at stage 10 and cultured until stage 19 before being measured for elongation. All images were taken under fluorescence. (C) RT-PCR analysis of Xbra transcripts from animal caps injected as in $A$, and cultured in the presence or absence of activin until stage 11 . Whereas dnFGFR completely blocked activin-induced mesoderm formation as measured by Xbra expression, Xbra was expressed in Xspry2 and $X$ spry2 $\Delta$ overexpressing caps. (-RT) whole embryo controls without reverse transcriptase. EF1 $\alpha$ was amplified as a loading control. $(D)$ Quantitation of the explant elongation assays from $A$ and $B .(n)$ number of animal caps examined; $(-)$ rounded explants; $(+)$ partial elongation; $(++)$ full extension.
(Fig. 5A). This could be indicative of a response to activin that was not coordinated into an integrated extension of the animal cap (Fig. 5A). Therefore, overexpression of Xspry2 in ectodermal explants resulted in a block of convergent extension while leaving the mesoderm formation intact.

To assess whether the inhibition of convergent extension is a property of the cells that normally undergo the process, we isolated dorsal marginal zone (DMZ) explants expressing a GFP lineage tracer together with Xspry2, dnFGFR, or the control HAV $\varnothing$ mRNA. The explants were then cultured until stage 19 and scored for the characteristic elongation of the involuting marginal zone. As shown in Figure 5B,D, the majority of control HAV $\varnothing$-injected embryos underwent extensive elongation. This process was largely inhibited by the dnFGFR and the Xspry2 molecules. As with the animal cap explants, we observed some weak morphogenetic movements in the presence of Xspry2 as compared to the dnFGFR indicating distinct consequences of overexpressing these two inhibitory molecules (Fig. 5B).

In summary, we have demonstrated using three inde- pendent assays that Xspry2 inhibits the convergent extension of mesodermal cells during gastrulation, both in the intact embryo and in explant cultures.

\section{Xsprouty2 does not inhibit FGF-mediated mesoderm induction or maintenance}

Having made the observation that overexpression of Xspry2 resulted in the inhibition of convergent extension movements in animal cap explants, but not affecting Xbra expression, we carried out in situ hybridization analysis to examine the consequence of Xspry2 expression on mesoderm induction and patterning in the embryo. Xbra is a member of the T-box family of transcription factors, which is expressed throughout the mesoderm during gastrulation (Smith et al. 1991). Moreover, Xbra is a direct target of FGF signaling during mesoderm induction, which requires the Ras/MAPK pathway, as Xbra expression was lost in embryos overexpressing dnRas (Fig. 6A). Surprisingly, the injection of Xspry2 or $X$ spry2 $\Delta$ mRNA did not have any effect on Xbra expression, suggesting that Xspry2 proteins did not inhibit Ras/ 
MAPK signaling. The mesoderm is patterned into domains, such as dorsal (characterized by Xnot expression), dorsolateral (characterized by XMyf5 expression), and

\section{A}

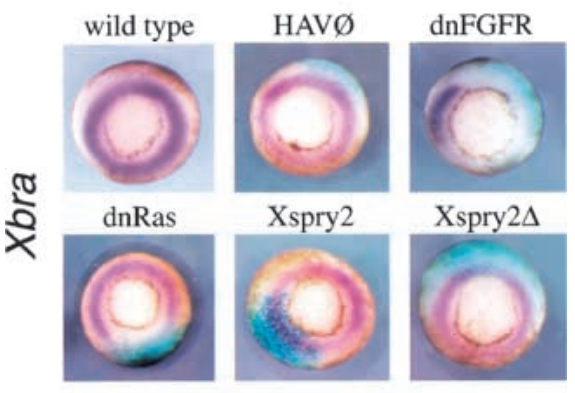

B

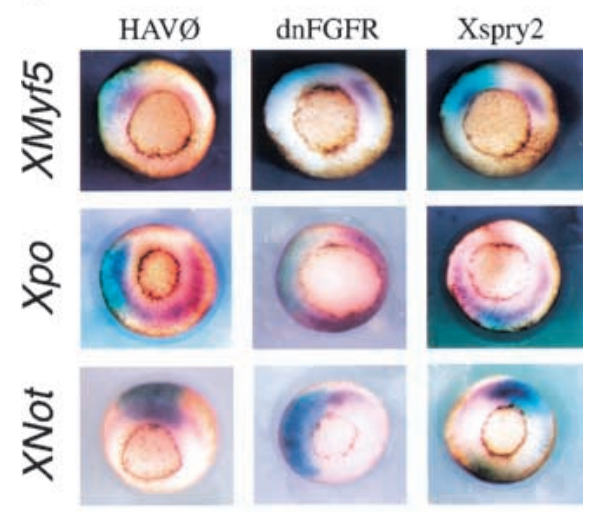

C

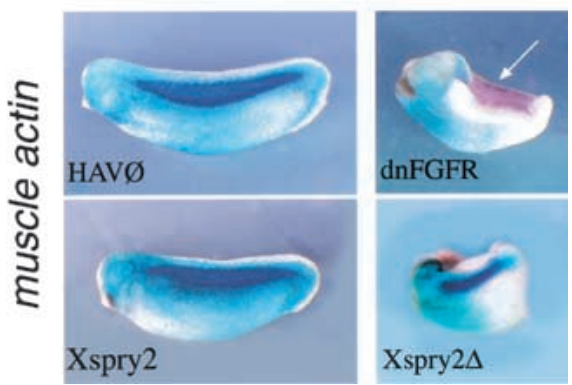

Figure 6. Xsprouty2 overexpression does not block mesoderm induction, patterning, or maintenance. $(A)$ Vegetal views of in situ hybridization (purple) to (A) Xbra and (B) Xmyf5, Xpo, and $X$ not at mid-gastrulation (stage 11). Embryos were injected at the four-cell stage into a dorsal blastomere with $1 \mathrm{ng}$ of mRNA along with $250 \mathrm{pg}$ LacZ mRNA. Embryos were stained for $\beta$-galactosidase activity (blue) as a lineage marker. $(C)$ In situ hybridization to muscle actin (purple) were performed on stage 25 embryos injected at the two-cell stage into a single blastomere with $1 \mathrm{ng}$ of either HAV $\varnothing$, dnFGFR, Xspry2, or Xspry2s together with $250 \mathrm{pg}$ of $\beta$-galactosidase mRNA (blue). Approximately $10 \%$ of Xspry2 and Xspry2 $\Delta$-injected embryos failed to close the blastopore yet retained muscle differentiation as assessed by coexpression of injected cells with muscle actin mRNA. Arrow indicates muscle actin expression on the noninjected side of dnFGFR embryos, which is absent of the $\beta$-galactosidase lineage tracer. ventral domains (characterized by Xpo expression). Injection of Xspry2 or Xspry2s did not alter the expression of any of these markers indicating that, in contrast to the dnFGFR or dnRas molecules, Xspry2 did not inhibit mesoderm induction or dorsoventral patterning (Fig. 6B; data not shown).

FGFR signaling has also been implicated in the subsequent maintenance of mesodermal cell fate during gastrulation (Kroll and Amaya 1996). Therefore, we have tested whether Xspry2-injected embryos expressed cardiac actin, a marker characteristic of the mesodermal derivative muscle. As shown in Figure 6C, cardiac actin was abundantly expressed in the somitic tissue of control HAV $\varnothing$-expressing embryos, coinjected with LacZ mRNA. The overexpression of dnFGFR, however, completely abrogated this actin expression on the injected side, leaving the untreated side intact. Importantly, although Xspry2 or Xspry2s overexpression led to the characteristic shortening of the A-P axis, cardiac actin was expressed appropriately along the dorsolateral domain of the embryo (Fig. 6C). This was the case both for the more mild truncations and the severe phenotype characterized by an open blastopore (Fig. 6C). Finally, FGF receptor function has been shown to be involved in the A-P patterning of the dorsal axis by controlling the expression of members of the caudal and posterior Hox gene families (Pownall et al. 1996, 1998). However, the posterior marker HoxA7 was expressed normally in $X$ spry or Xpsry2s-overexpressing embryos at the early neurula stages, in contrast to the absence of gene expression observed in dnFGFR-injected embryos (data not shown). Thus, in stark contrast to the inhibition of FGF signaling mediated by dnFGFR, Xspry 2 did not exert any significant effect on development by inhibiting mesoderm induction, patterning, or maintenance.

Xsprouty2 functions intracellularly in the FGF signaling pathway to block calcium mobility but not MAPK phosphorylation

The mechanism by which Spry inhibits RTK signaling is unclear (Hacohen et al. 1998; Casci et al. 1999; Kramer et al. 1999; Reich et al. 1999|. Therefore, we have attempted to dissect the biochemical pathways by which Xspry2 functions by using a convenient assay for FGF receptor function in Xenopus oocytes, based on the mobilization of intracellular calcium stores within minutes of FGF addition (Musci et al. 1990). Briefly, Xenopus oocytes are injected with FGF receptor mRNA and allowed to translate the receptor for a couple of days. The oocytes are loaded with ${ }^{45} \mathrm{Ca}^{2+}$, washed extensively, and assayed for the release of ${ }^{45} \mathrm{Ca}^{2+}$ into the medium before and after FGF addition. Xenopus oocytes expressing FGFR with a 100 -fold excess of HAV $\varnothing$ produced with the addition of FGF2, a rapid efflux of $\mathrm{Ca}^{2+}$ from the cell, which was blocked by the coexpression of dnFGFR (Fig 7A; Amaya et al. 1991). This FGF2 dependent $\mathrm{Ca}^{2+}$ response was also inhibited by the coexpression of a 100 fold excess of either Xspry2 or Xspry2s with the FGFR (Fig. 7A). Thus, 
A
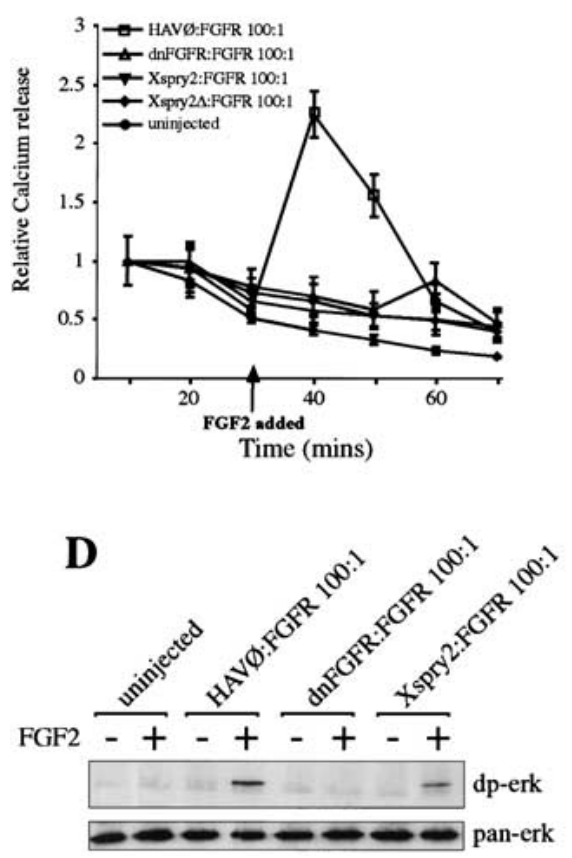

B

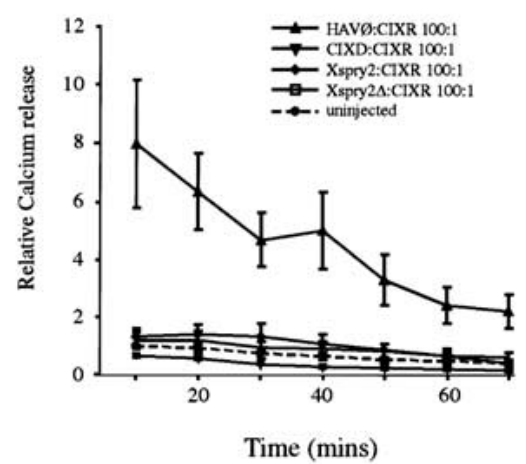

$\mathbf{E}$

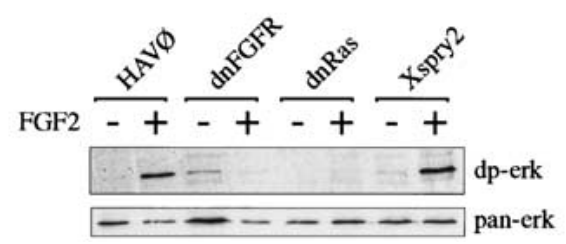

C
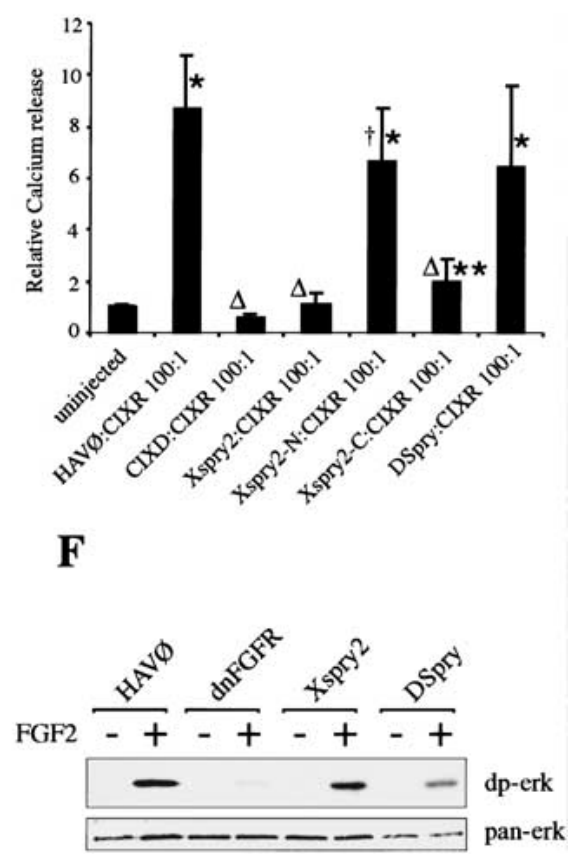

Figure 7. Xsprouty2 proteins function as intracellular inhibitors of the FGF signaling pathway. (A) Xspry2 proteins block FGF signaling. FGF-stimulated calcium efflux from oocytes was measured after injection of $250 \mathrm{pg}$ of Xenopus FGFR1 (FGFR) and 25 ng of the indicated mRNA (100-fold excess). Oocytes were loaded with ${ }^{45} \mathrm{Ca}^{2+}$ and the media collected at 10 -min intervals, before and after the addition of FGF2 (100 ng/ml) and subjected to scintillation counting. All points represent the mean of at least nine wells (10 oocytes per well), derived from three independent experiments. For standardization, the counts obtained in the first 10 min for each condition was given a value of 1 and all other points are shown relative to the counts measured for each condition in the first 10 min. Error bars indicate \pm S.E.M. (B) Xspry2 functions downstream of the FGFR. Oocytes were injected as in $A$ with 250 pg of the constitutively active RTK, CIXR, and the indicated mRNA at $25 \mathrm{ng}$. CIXD is a dominant negative form of CIXR; CIXR constitutively dimerizes and signals in the absence of ligand, as seen by the FGF-independent calcium efflux. For standardization, all values are shown relative to the counts obtained from uninjected oocytes in the first $10 \mathrm{~min}$. (C) DSpry does not inhibit $\mathrm{Ca}^{2+}$ signaling in oocytes, but the carboxyl terminus of Xspry2 does inhibit $\mathrm{Ca}^{2+}$ signaling. Oocytes were injected and assayed as in $B$, except that only the relative ${ }^{45} \mathrm{Ca}^{2+}$ release for the first $10 \mathrm{~min}$ is shown. Error bars indicate \pm S.D. ${ }^{\star} P<0.001$ and ${ }^{\star \star} P<0.01$ compared to uninjected. ${ }^{\Delta} P<0.001$ and ${ }^{\dagger} P<0.05$ compared to HAV $\varnothing$ :CIXR. $(D)$ Xspry2 does not inhibit FGF-dependent MAPK activation in ovo. Oocytes were injected and cultured as in $A$, before being incubated with or without FGF2 $(100 \mathrm{ng} / \mathrm{ml})$ for 15 min. Protein extracts were then analyzed for activated (dp-erk) and total (pan-erk) MAPK levels. (E) MAPK in animal cap explants is not sensitive to Xspry2 overexpression. Animal caps injected with the indicated mRNA were incubated for 15 min with or without FGF2 before being subjected to immunoblotting as in $D .(F)$ DSpry does not inhibit MAPK activation in FGF-treated animal caps. Animal caps were injected with the indicated mRNAs and treated as in $E$.

Xspry2 functioned directly to block a physiological response of the activated FGFR. To test whether Xspry2 acted intracellularly, we expressed a constitutively active FGFR, termed CIXR (Amaya, 1992). CIXR consists of the dimerization domain of the bacteriophage $\lambda$ repressor fused to the FGFR transmembrane and intracellular domains. This construct is expected to be constitutively active as receptor dimerization is the essential step in activating the endogenous receptor; however, as the protein lacks the ligand-binding domain of the FGFR, it would not be responsive to specific extracellular signals. As expected, CIXR produced a constitutive $\mathrm{Ca}^{2+}$ response when injected into oocytes, which could be blocked by CIXD, a dominant negative version of CIXR, truncated in the intracellular domain in a manner analogous to that of dnFGFR (Fig. 7B,C). Coexpression of either Xspry2 or Xspry2 $\Delta$ with CIXR resulted in the inhi- bition of the $\mathrm{Ca}^{2+}$ mobilization. Thus, both proteins act independently of the FGFR extracellular domain (Fig. $7 \mathrm{~B}, \mathrm{C})$.

Finally, the calcium efflux assay provided us with an opportunity to dissect the functional domains of Xspry2. To commence this process we have separated the evolutionarily less conserved amino terminus (Xspry2-N; amino acids 1-174) from the highly conserved carboxyl terminus (Xspry2-C; amino acids 176-314). Neither protein had the ability to inhibit convergent extension movements in animal cap explants or in whole embryo assays, indicating that the functional protein requires motifs from both regions of the gene (see Fig. 5D; data not shown). However, the Xspry2-C motif alone was able to efficiently inhibit the calcium efflux in the oocyte assay (Fig. 7C). The ability of Xspry2-C protein to function in the oocyte assay but not in animal cap explants 
may indicate that blocking the calcium pathway is not sufficient to inhibit gastrulation movements. Alternatively, it is also possible that a partially active protein can, when highly expressed in the oocyte, retain some function but not be able to do so in the more complex environment of the embryo.

To determine whether Xspry2 could directly inhibit Ras/MAPK signaling, we assayed for the activation of MAPK by immunoblotting using an antibody specific for the diphosphorylated active form of MAPK (dp-erk). As expected FGFR-injected oocytes displayed a rapid FGF2dependent activation of MAPK, a response that was completely blocked by dnFGFR (Fig. 7D). Importantly, this FGF-dependent phosphorylation of MAPK was also detected in the oocytes overexpressing Xspry2 (Fig. 7D). Hence, Xspry2 blocked FGF-dependent calcium mobilization in oocytes but had no obvious effect on MAPK activation. However, we found that the MAPK pathway in Xenopus oocytes is not completely analogous to that in the embryo, as the dnRas construct does not block MAPK phosphorylation in ovo (data not shown). Therefore, to compare Xspry2 function to that of the dnRas, we assayed for MAPK activation in animal caps treated with FGF2. We found that animal caps expressing the control HAV $\varnothing$ construct showed a rapid FGF-dependent activation of MAPK. As expected, the dnFGFR and dnRas molecules completely inhibited the FGF-dependent activation of MAPK, whereas the injection of Xspry2 had no overt effect on MAPK activation (Fig. 7E). In summary, these experiments showed that Xspry2 acts downstream of the activated FGFR to inhibit calcium mobilization, but does not block Ras/MAPK activity.

The inability of Xspry2 to impair MAPK activation in oocytes or animal cap explants is in contrast to observations in Drosophila where Spry (DSpry) blocks steadystate dp-erk levels (Reich et al. 1999). To assess whether this is an intrinsic difference between the Drosphila and Xenopus Spry proteins or a difference in FGFR signaling between the two organisms, we have expressed DSpry in oocytes and animal cap explants and assayed for MAPK activation, activin-induced convergent extension movements, and calcium mobilization. In the animal cap assay DSpry had an identical effect to Xspry2 in blocking convergent extension movements (see Fig. 5D), while leaving MAPK activation largely intact (Fig. 7F). However, in the oocyte calcium release assay, DSpry, in contrast to Xspry2, was unable to inhibit the calcium efflux mediated by the constitutively active CIXR construct (Fig. 7C). Thus, DSpry provides us with a reagent that discriminates between the functions of Spry proteins in inhibiting calcium mobilization and convergent extension, suggesting that these processes may not be causally related.

\section{Discussion}

The regulation of RTK signaling pathways has long been considered essential for normal development, but the mechanisms by which the multitude of ligands, receptors and potential pathways are controlled has remained an intractable problem (Simon 2000). FGFs are known to signal through their cognate receptors and the Ras/ MAPK pathway to initiate mesoderm induction both in embryos and animal cap explants (for review, see Gotoh and Nishida 1996). However, the physiological roles of the other potential FGF-initiated signaling pathways as well as the role of FGF after mesoderm induction have proved difficult to examine. We have identified Xspry2 as an intracellular antagonist of the FGF pathway during Xenopus gastrulation. Xspry2 expression is induced in the early gastrula in an FGF-dependent manner and directly inhibits some aspects of FGF-dependent intracellular signaling, such as calcium mobilization, while not affecting the MAPK pathway. As a consequence, Xspry2 overexpression leads to normal mesoderm induction and patterning but severely inhibits the establishment of the embryonic A-P axis through convergent extension. Thus, Xsprouty2 is the first protein identified that inhibits aspects of the FGF response such as gastrulation morphogenesis, while leaving the inductive and patterning processes intact.

Xsprouty2 is both a component and a downstream target of the FGF signaling pathway

Previous studies in Drosophila and in vertebrates have noted the coincidence of sprouty expression and active sites of FGF signaling (Hacohen et al. 1998; Minowada et al. 1999; Tefft et al. 1999; Chambers and Mason 2000). Moreover, the implantation of beads coated with either FGF4 or FGF8 into chick embryos (Minowada et al. 1999; Chambers et al. 2000) or the overexpression of branchless (Bnl), the Drosophila FGFR1 homolog induces spry expression (Hacohen et al. 1998). This transcriptional response is not limited to FGF-dependent signaling as the Drosophila EGFr also induces spry (Casci et al. 1999; Kramer et al. 1999; Reich et al. 1999). These results have given rise to the hypothesis that sproutys are immediate targets of RTK signaling. In Xenopus studies using the dp-erk antibody, which recognizes activated MAPK, indicate that the principle sites of active RTK signaling through the Ras pathway during early development requires FGFR signaling (Christen and Slack 1999). In agreement with this observation, the expression of Xspry2 from the late blastula to tail bud stages closely mimics that of MAPK activity (Fig. 2A; Christen and Slack 1999). Moreover, Xspry2 is expressed in a manner similar to XFGF8 in the developing neural structures, sensory organs, and tail bud (Fig. 2B,C). This matching pattern of XFGF8 and spry2 expression also occurs in the mouse and chick (Minowada et al. 1999; Chambers et al. 2000). By using the dnFGFR and dnRas constructs to specifically block the FGF and Ras/MAPK pathway, we show that Xspry2 depends on an intact FGFR/Ras/ MAPK signaling cascade for its expression.

Genetic studies in Drosophila indicate that Spry is an inhibitor of the FGF pathway, but little biochemical evidence has been provided to indicate whether Spry acts directly to regulate RTK signal transduction. Indeed, the genetic data so far presented provides a conflicting pic- 
ture, as Spry was initially proposed to function as an extracellular antagonist, inhibiting FGF signaling in neighboring cells. However, when Spry was also identified as a negative regulator of EGFr signaling during Drosophila development, its mode of activity was reassessed and Spry was proposed to function intracellularly to inhibit the Ras pathway (Casci et al. 1999; Reich et al. 1999). There is further debate in the literature as to whether Spry inhibits the pathway by blocking Ras activation (Casci et al. 1999), or alternatively functions downstream by inhibiting Raf (Reich et al. 1999). In addition to the genetic data, results from cell transfection experiments suggested that Spry associates with the inner surface of the plasma membrane, and may bind two components of the Ras/MAPK pathway, Gap1 and Drk (Drosophila Ras-GAP and Grb2; Casci et al. 1999). Our data, using the oocyte calcium efflux assay, which relies on the rapid (within $10 \mathrm{~min}$ ) ligand-dependent mobilization of intracellular $\mathrm{Ca}^{2+}$ stores, provides the first direct evidence that sprouty proteins can block an aspect of RTK signaling. These experiments also strongly support the hypothesis that Xspry2 acts independently of the FGFR extracellular domain as it inhibits signaling elicited by a constitutively active FGFR that contains no FGFR extracellular sequences.

\section{Multiple signals arising from the FGF receptor during development}

Although a wealth of studies have been carried out in cell culture systems on the signals that emanate from the FGFR and their downstream consequences, most of the experiments examining FGFR-mediated events in developmental or genetic model systems has concentrated on the Ras/MAPK pathway. The activation of the MAPK pathway in naive Xenopus animal cap explants leads to the induction of mesoderm tissue (Umbhauer et al. 1995). Conversely, the elimination of Ras/MAPK activity, by a variety of dominant negative molecules, prevents mesoderm formation (for review, see Gotoh and Nishida 1996). Thus. Ras/MAPK signals are essential for the establishment of mesodermal tissue. However, a number of proteins are known to associate with activated RTKs such as the FGFR (for review, see Pawson and Saxton 1999). These include the adapter molecules Nck and Grb2 (Gupta and Mayer 1998), PLC- $\gamma 1$, PI3K, the tyrosine phosphatase shp2, and the Ras-GTPase-activating protein (GAP; Ryan et al. 1998). These molecules are capable of activating a number of signaling pathways besides those involving Ras/MAPK. Phosphorylated tyrosine 766 (Y766) of the FGFR has high affinity for PLC- $\gamma 1$ and is essential for phosphatidylinositol hydrolysis, which results in the activation of protein kinase $\mathrm{C}$ (PKC) and the mobilization of intracellular calcium stores, which in turn can influence $\mathrm{Ca}^{2+}$-sensitive transcription factors. Indeed, in Xenopus and zebrafish $\mathrm{Ca}^{2+}$ transients are associated with gastrulation (Slusarski et al. 1997). The significance of this signal for gastrulation has not been established as the overexpression of a PDGFR/FGFR chimeric molecule containing the Y766F mutation (which cannot be phosphorylated) in Xenopus animal caps revealed that mesoderm induction could occur in the absence of this signal (Muslin et al. 1994). In addition, phosphopeptide mapping has revealed six further phosphorylated tyrosines on the activated FGFR1, of which two are required for the intrinsic kinase activity of the dimerized receptor and the remaining four are dispensable for FGF-mediated mitogenesis, MAPK activation, and differentiation in PC12 cells (Mohammadi et al. 1996). Thus, the function of the factors that associate with the FGFR1 and the signaling pathways arising from them remains unclear. We have shown here that Xspry2 blocks calcium mobilization induced by FGF in oocytes. Moreover, unlike dnRas, Xspry2 does not block mesodermal gene expression and MAPK activation. Therefore, we propose that Xspry2 impinges on an FGF-dependent signaling pathway that is independent of Ras. In the future it will be important to dissect further the pathway by which Xspry2 exerts its effects by using specific mutations in the FGFR1 intracellular domain.

The biochemical data presented here on the role of Xspry2 in $\mathrm{Ca}^{2+}$ mobilization differs from those that indicate an involvement of Spry in Ras signaling in the fly (Casci et al. 1999; Kramer et al. 1999; Reich et al. 1999). Although the exact placement of Drosophila Spry in the pathway is unclear, the general conclusion is that Spry inhibits Ras signaling. Our demonstration that Xspry2 does not have a major impact on MAPK activation would suggest that the vertebrate homologs have evolved a distinct function in FGFR-dependent signaling. In fact, Xspry2 and DSpry show virtually no sequence conservation at the amino acid level, outside the carbox-terminal cysteine-rich domain (49\% identity, overall $27 \%$ ). In addition, the mammalian genomes contain at least four spry homologs that could have evolved more diverse functions than the single Drosophila gene. This is supported by the differing abilities of the Xspry2 and DSpry to block $\mathrm{Ca}^{2+}$ mobilization in the oocyte assay (Fig. $7 \mathrm{C}$ ). However, both molecules were equally capable of blocking convergent extension movements in animal cap explants while leaving MAPK activation intact, indicating that at least this property of the proteins has been conserved through evolution. It is worth noting that in contrast to the data presented here, only steady-state levels of Drosophila MAPK activation were measured, and no alternative RTK-induced pathways were tested. Moreover, RTK signaling networks have been shown in a number of systems to maintain extensive pathway crosstalk. Thus, a linear approach to understanding the role of Spry proteins in RTK signaling may be misleading.

\section{Xsprouty2 inhibits gastrulation movements without affecting mesoderm induction and maintenance}

A long-standing problem in understanding the potential role for FGF signaling in gastrulation movements has been the requirement of the pathway in mesoderm induction. Inhibiting the FGF/Ras/MAPK signal transduction pathway results in the failure of mesoderm induction and as such the subsequent interpretation of gastru- 
lation defects in the absence of FGF activity is not possible (Amaya et al. 1991). However, diverse evidence from a number of other systems does implicate FGF in the morphogenesis of gastrulation. $f g f r 1^{-/-}$and $f g f 8^{-/-}$ mice have severe gastrulation defects, including abnormalities in the migration of cells within the primitive streak (Deng et al. 1994; Yamaguchi et al. 1994; Ciruna et al. 1997; Sun et al. 1999). Furthermore, mosaic analyses with $f g f r 1^{-/-}$embryonic stem cells indicate that the primary defect of fgfr1-deficient cells may be in their inability to traverse the primitive streak (Ciruna et al. 1997). These data are supported by mutations in the Drosophila FGFR2 homolog Heartless (HtI), in which the invaginated mesoderm fails to migrate in a dorsolateral direction from the ventral midline, resulting in a reduction in cardiac, visceral, and dorsal muscle cell fates (Beiman et al. 1996; Gisselbrecht et al. 1996). In Xenopus experiments have revealed that FGF2 can induce cell motility, lamellipodia formation, and cellular polarization in gastrula stage cells (Wacker et al. 1998). Together, these studies indicate a conserved role for FGFR signaling in the mesoderm morphogenesis.

The Xspry2 overexpression studies presented here provide the first instance whereby the role of FGF signaling in Xenopus mesoderm induction and maintenance has been separated from its role in morphogenesis. Xspry2 overexpressing embryos have normal mesoderm induction and patterning, but fail to undergo the gastrulation movements essential for the establishment of the body plan. This raises the question of why an inhibitor of gastrulation is expressed in the very cells that normally undergo such movements? The answer probably lies in the fact that the cell motility that drives convergent extension movements must be exquisitely coordinated. It is initiated laterally and then progresses medially (Shih and Keller 1992). As a result convergent extension progresses as a wave from the lateral edges of the dorsal mesoderm toward the midline. This coordination is likely to involve molecules that can regulate both the timing and progression of these movements. We propose that Xspry2 is one such molecule. Therefore, when Xspry2 is overexpressed, this coordination is lost and the process of convergent extension is disrupted.

Although our data suggest that Xspry2 inhibits convergent extension, the coordination of this process must also involve signals that promote cell movements. Recent evidence suggests that the noncanonical Wnt pathway may provide this other signal. The zebrafish silberblick (slb) locus encodes the Wnt11 protein, which when mutated results in a gastrulation defect, as the $s 1 b^{-/-}$ cells fail to undergo convergent extension of the axial mesoderm. Moreover, the $s 1 b$ mutant phenotype is rescued by a truncated form of disheveled that does not signal through the canonical Wnt pathway (Heisenberg et al. 2000). In support of this data, the overexpression of an inhibitory form of disheveled (Xdd1), the wild-type frizzled 7 receptor, or a dominant negative Xwnt11 in Xenopus embryos results in a similar defect in gastrulation (Djiane et al. 2000; Tada and Smith 2000; Wallingford et al. 2000). In all these cases the defect in the con- vergent extension of the dorsal mesoderm is independent of its induction and specification (Sokol 1996). Thus, the consequences of Xspry2 overexpression or blocking aspects of Wnt11 signaling are similar. It may be noteworthy that, although we have shown that Xspry2 inhibits $\mathrm{Ca}^{2+}$ signaling, the noncanonical Wnt pathway promotes the same process (Slusarski et al. 1997). However, the lack of correlation between the ability of DSpry and Xspry2-C to inhibit convergent extension and $\mathrm{Ca}^{2+}$ mobilization would argue against a role for $\mathrm{Ca}^{2+}$ signaling per se in coordinating the cell movements of gastrulation. Finally, it is known that Xwnt11 is regulated by Xbra (itself a target of the FGF pathway) and therefore, is a downstream consequence of FGFR activation (Tada and Smith 2000). Therefore, it has been proposed that the role of FGF in gastrulation is an indirect result of the induction of Xwnt11 by Xbra (Sokol 1999). The data presented here enables us to propose the following extension to this model where FGF has both indirect (through Xbra and Xwnt11) and direct (through Xspry2) roles in gastrulation (Fig. 8).

During the late blastula stage signaling through the FGFR by the Ras/MAPK pathway induces expression of mesodermal target genes including Xbra. Xbra is a transcriptional activator whose targets include Xwnt11 (Tada and Smith 2000) and XeFGF (Isaacs et al. 1994). XeFGF then functions with Xbra in an autocatalytic loop to reinforce mesoderm fate (Isaacs et al. 1994; SchulteMerker and Smith 1995). Another target of Ras/MAPK signaling is Xspry2, which functions to inhibit some aspects of FGFR signaling that are independent of the Ras/ MAPK pathway and ultimately control the convergent extension movements of gastrulation. Therefore, Xspry2 provides a mechanism by which the FGFR can provide distinct signals to coordinate the processes of mesoderm induction and gastrulation morphogenesis, potentially through the interaction with the noncanonical Wnt pathway. Understanding the multiple levels of interactions between FGF signaling pathways and other signaling pathways, such as the noncanonical Wnts, will be essential for understanding how the coordination of mesoderm induction, maintenance, and convergent extension is achieved during gastrulation.

\section{Materials and methods}

Isolation of Xsprouty2 cDNAs and plasmid constructs

The Xenopus laevis homologs of human SPROUTY2 were isolated by screening a head library (Hemmati-Brivanlou et al. 1991) with the carboxy-terminal region of the hSPRY2 ORF (nucleotides 423-940) (cDNA received from Genome Systems; GenBank accession no. R55258). The full-length Xsprouty2 genes (GenBank accession nos. AF331824 and AF331825) were amplified using the primer combination 5'-GGAATTCCATG GAGACGAGAGTA and 5'-GCTCTAGACTATGTTGGTTT TTC and cloned into the EcoRI and XbaI sites of pCS2 +. The plasmids Xspry2-N and Xspry2-C consist of amino acids 1-174 and 176-314 respectively. Full length Drosophila spry (Hacohen et al. 1998) was cloned into the EcoRI/XbaI sites of pCS2 + to generate the expression construct pDSpry. The constitutively 


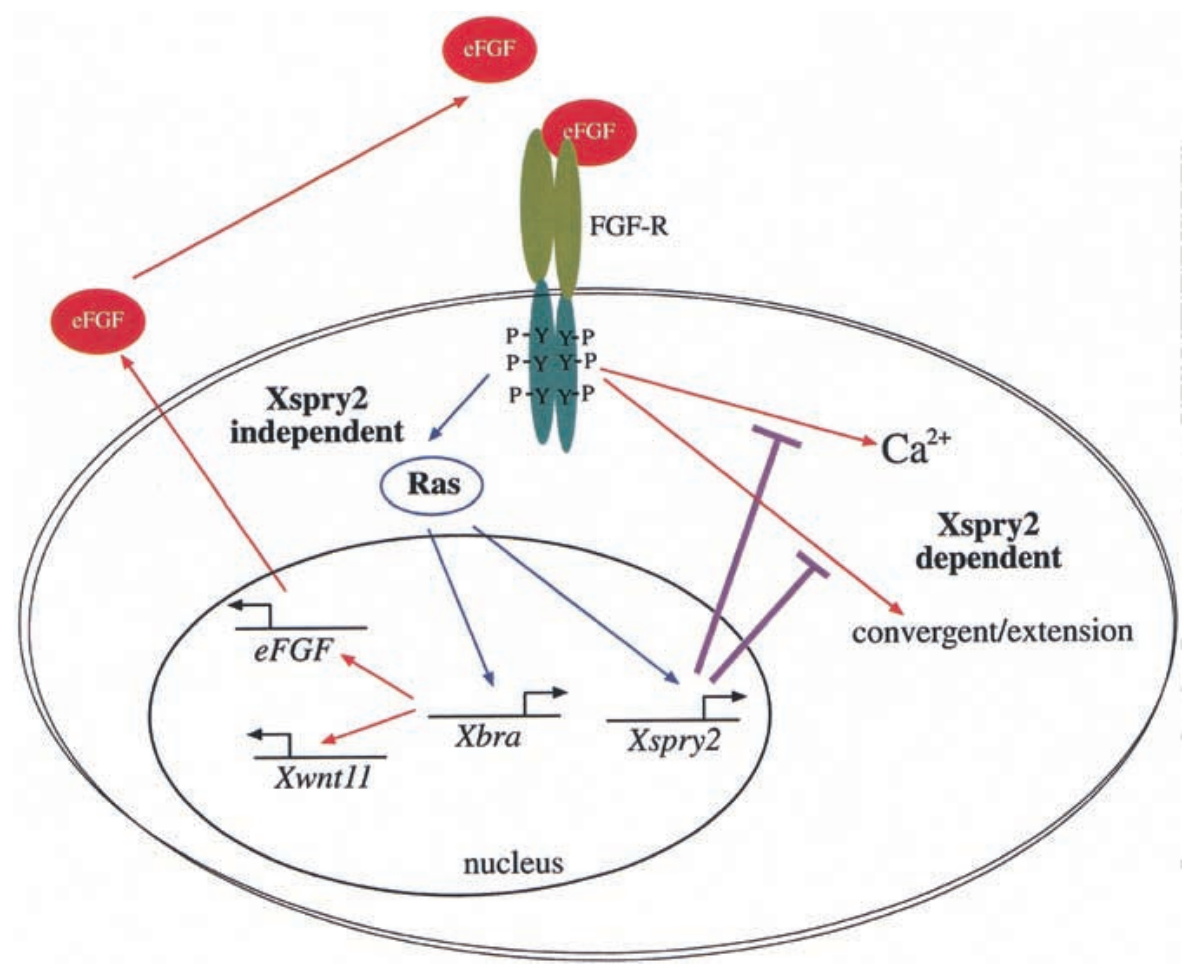

Figure 8. Model for the function of Xsprouty2 in Xenopus gastrulation. At least two signaling pathways emanate from the FGFR during gastrulation: a Xsprouty2-insensitive signal through the Ras/MAPK pathway results in mesoderm induction/maintenance as well as Xsprouty2, Xbra, and indirectly eFGF and Xwnt11 expression. The second Xsprouty2-sensitive pathway results in Ca ${ }^{2+}$ mobilization and convergent extension, two processes known to require Xwnt11 signaling. Thus, Xsprouty2 functions to coordinate the distinct roles of the FGFR in mesoderm induction and morphogenesis. See discussion for further details.

active FGFR1 construct pCIXR and its dominant negative version pCIXD have been described previously (Amaya 1992). pCIXR consists of the first 51 amino acids of the chicken FGFR1 (Lee et al. 1989), including the sequences encoding the signal peptide, fused to amino acids $97-237$, encoding the dimerization domain of the bacterial $\lambda$ repressor and amino acids 334 1211 from the Xenopus FGFR1 (Musci et al. 1990), including the transmembrane and cytoplasmic domains. pCIXD, lacks the cytoplasmic domain of the CIXR in a position identical to that of dnFGFR (XFD/Xss; Amaya et al., 1991).

\section{mRNA synthesis for microinjection}

Capped mRNA was synthesized using the Ambion Message Machine kit and SP6 polymerase. The expression constructs pXspry2, pXspry2 $\Delta$, and pDSpry were linearized with NotI, pXspry2-N, and pXspry2-C with Asp718 and pCIXR and pCIXD were digested with SacI. The plasmids pHAV $\varnothing$ (nonfunctional FGFR), XFD/Xss (dnFGFR), p21 (Asn17) $)^{\text {Ha-ras }}$ (dnRas), pnucßgal (nuclear localized $\beta$-galactosidase), pXFR $\Delta 3^{\prime}$ (Xenopus FGFR1), and pGAP43-GFP (membrane tethered eGFP) have been previously described (Amaya et al. 1991, 1993; Smith and Harland 1991; Whitman and Melton 1992; Amaya et al. 1993; Kim et al. 1998).

\section{RNA isolation and RT-PCR analysis}

Total RNA was prepared from embryos using the Trizol reagent (GIBCO-BRL). Random primed cDNAs were generated from 3 $\mu \mathrm{g}$ of each RNA preparation using the SUPERSCRIPT-II reverse transcriptase (GIBCO-BRL). The PCR was performed using 0.15 ng of each primer, $0.2 \mathrm{mM}$ dNTPs, $5 \mathrm{nl}$ of $\left[{ }^{32} \mathrm{P}\right] \mathrm{dATP}, 1.5 \mathrm{mM}$ $\mathrm{MgCl}_{2}$, and 2 units of Taq polymerase (Roche Molecular Biochemicals). PCR amplification was performed for 20-23 cycles and determined to be in the linear range for each primer pair.

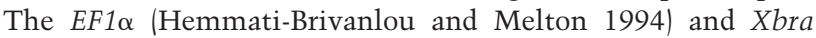
(Henry et al. 1996) primers have been previously described.

\section{Embryo injections and manipulations}

Embryos were obtained and injected as previously described (Amaya et al. 1993). Lineage tracing experiments were carried out by injecting a single dorsal blastomere at the four-cell stage with $25 \mathrm{ng}$ of RLDX and the adjacent dorsal blastomere with 1 ng of Xspry2/GAP43-GFP or HAVØ/GAP43-GFP mRNA (10:1 ratio). Animal caps were excised from stage 8 embryos and incubated in $0.4 \times$ MMR, or $1 \times$ MMR for immunoblotting. Recombinant human activin A (10 ng/ml; R\&D Systems) was added as appropriate and caps were incubated until stage 11 for molecular analysis or stage 18 and scored for elongation. DMZ explants were excised at stage 10 and cultured in $0.4 \times$ MMR until stage 19.

\section{In situ hybridization and lineage tracing}

In situ hybridization was carried out according to the method of Harland (1991). An RNA in situ probe against Xspry2 was generated by linearizing with BamHI and transcribing with $\mathrm{T} 7$. The Xbra, cardiac actin (AC100), Xpo (Amaya et al. 1991, 1993), Xmyf5, XNot (von Dassow et al. 1993), and FGF8 (Christen and Slack 1997) probes are as previously reported. 
Nutt et al.

${ }^{45} \mathrm{Ca}^{2+}$ efflux assay and oocyte injections

Oocytes were obtained and microinjected as described (Amaya et al. 1991). After $48 \mathrm{~h}$ of incubation in modified Barth's saline (MBSH) at $16^{\circ} \mathrm{C}$, healthy oocytes were assayed for FGF-induced ${ }^{45} \mathrm{Ca}^{2+}$ efflux essentially as reported (Musci et al. 1990). Oocytes were washed in $\mathrm{Ca}^{2+}$-free $\mathrm{MBSH}$ and transferred 10 per well in triplicate into 24 -well plates. ${ }^{45} \mathrm{Ca}^{2+}$ was added at a final concentration of $50 \mu \mathrm{Ci} / \mathrm{ml}$ and loaded for $3 \mathrm{~h}$ at $16^{\circ} \mathrm{C}$. Oocytes were then washed extensively in MBSH before culturing in a final volume of $1 \mathrm{ml}$. Aliquots were then removed at $10-\mathrm{min}$ intervals and replaced with an equal volume of MBSH. FGF2 was added after $30 \mathrm{~min}$ at a final concentration of $100 \mathrm{ng} / \mathrm{ml}$. Medium collected after seven time points was then subjected to scintillation counting in Ecolume (ICN). Variation between the two groups was tested using the Student's $t$ test. $P$ values $<0.05$ are indicated.

\section{Immunoblotting}

Western blotting was carried out as described (Amaya et al. 1993), with the exception that $50 \mathrm{mM} \mathrm{NaF}$ and $10 \mathrm{mM}$ sodium orthovanadate were added to the lysis buffer. Blots were incubated with 1/10,000 mouse anti-dperk (clone MAPK-YT, Sigma) or 1/2000 mouse anti-pan erk (clone 16, Transduction laboratories).

\section{Acknowledgments}

We thank N. Papalopulu for help with the explants and S. Bramley for cloning the Xspry2 mutants. We are grateful to H. Isaacs, M. Freeman, M. Whitman, J. Gurdon, R. Harland, and D. Kimelman for the gifts of reagents and N. Papalopulu, M. Gomperts, O. Bronchain, A. Philpott, W. Harris, and members of the Harris/Holt laboratories for helpful discussions. This work was supported by European Molecular Biology Organization and Human Frontier Science Program long-term fellowships (S.L.N.), a Burroughs Wellcome Hitchings-Elion Fellowship (K.S.D.), a program grant from the Medical Research Council (C.E.H.), and a Wellcome Trust Senior Research Fellowship (E.A.).

The publication costs of this article were defrayed in part by payment of page charges. This article must therefore be hereby marked "advertisement" in accordance with 18 USC section 1734 solely to indicate this fact.

\section{References}

Amaya, E., Musci, T.J., and Kirschner, M.W. 1991. Expression of a dominant negative mutant of the FGF receptor disrupts mesoderm formation in Xenopus embryos. Cell 66: 257-270.

Amaya, E. 1992. FGF signalling in early Xenopus development. Phd Thesis. University of California, San Francisco.

Amaya, E., Stein, P.A. Musci, T.J., and Kirschner, M.W. 1993. FGF signalling in the early specification of mesoderm in Xenopus. Development 118: 477-487.

Beiman, M., Shilo, B.Z., and Volk, T. 1996. Heartless, a Drosophila FGF receptor homolog, is essential for cell migration and establishment of several mesodermal lineages. Genes \& Dev. 10: 2993-3002.

Carballada, R., Yasuo, H., and Lemaire, P. 2001. Phosphatidylinositol-3 kinase acts in parallel to the ERK MAP kinase in the FGF pathway during Xenopus mesoderm induction. Development 128: 35-44.
Casci, T., Vinos, J., and Freeman, M. 1999. Sprouty, an intracellular inhibitor of Ras signaling. Cell 96: 655-665.

Chambers, D. and Mason, I. 2000. Expression of sprouty2 during early development of the chick embryo is coincident with known sites of FGF signalling. Mech. Dev. 91: 361-364.

Chambers, D., Medhurst, A.D. ,Walsh, F.S., Price, J., and Mason, I. 2000. Differential display of genes expressed at the midbrain-hindbrain junction identifies sprouty2: An FGF8inducible member of a family of intracellular FGF antagonists. Mol. Cell Neurosci. 15: 22-35.

Christen, B. and Slack, J.M. 1997. FGF-8 is associated with anteroposterior patterning and limb regeneration in Xenopus. Dev. Biol. 192: 455-466.

. 1999. Spatial response to fibroblast growth factor signalling in Xenopus embryos. Development 126: 119-125.

Ciruna, B.G., Schwartz, L., Harpal, K., Yamaguchi, T.P., and Rossant, J. 1997. Chimeric analysis of fibroblast growth factor receptor-1 (Fgfr1) function: A role for FGFR1 in morphogenetic movement through the primitive streak. Development 124: 2829-2841.

Cornell, R.A. and Kimelman, D. 1994. Activin-mediated mesoderm induction requires FGF. Development 120: 453-462.

de Maximy, A.A., Nakatake, Y., Moncada, S., Itoh, N., Thiery, J.P., and Bellusci, S. 1999. Cloning and expression pattern of a mouse homologue of Drosophila sprouty in the mouse embryo. Mech. Dev. 81: 213-216.

Deng, C.X., Wynshaw-Boris, A., Shen, M.M., Daugherty, C., Ornitz, D.M., and Leder, P. 1994. Murine FGFR-1 is required for early postimplantation growth and axial organization. Genes \& Dev. 8: 3045-3057.

Djiane, A., Riou, J., Umbhauer, M., Boucaut, J., and Shi, D. 2000. Role of frizzled 7 in the regulation of convergent extension movements during gastrulation in Xenopus laevis. Development 127: 3091-3100.

Fambrough, D., McClure, K., Kazlauskas, A., and Lander, E.S. 1999. Diverse signaling pathways activated by growth factor receptors induce broadly overlapping, rather than independent, sets of genes. Cell 97: 727-741.

Gisselbrecht, S., Skeath, J.B., Doe, C.Q., and Michelson, A.M. 1996. heartless encodes a fibroblast growth factor receptor (DFR1/DFGF-R2) involved in the directional migration of early mesodermal cells in the Drosophila embryo. Genes \& Dev. 10: 3003-3017.

Gotoh, Y. and Nishida, E. 1996. Signals for mesoderm induction. Roles of fibroblast growth factor $(\mathrm{FGF}) /$ mitogen-activated protein kinase (MAPK) pathway. Biochim. Biophys. Acta 1288: F1-F7.

Gupta, R.W. and Mayer, B.J. 1998. Dominant-negative mutants of the SH2/SH3 adapters Nck and Grb2 inhibit MAP kinase activation and mesoderm-specific gene induction by eFGF in Xenopus. Oncogene 17: 2155-2165.

Hacohen, N., Kramer, S., Sutherland, D., Hiromi, Y., and Krasnow, M.A. 1998. sprouty encodes a novel antagonist of FGF signaling that patterns apical branching of the Drosophila airways. Cell 92: 253-263.

Harland, R.M. 1991. In situ hybridization: an improved whole mount method for Xenopus embryos. In Methods in Cell Biology. (eds B.K. Kay and H.B. Peng), pp. 685-695. San Diego, Academic Press

Heisenberg, C.P., Tada, M., Rauch, G.J. Saude, L., Concha, M.L., Geisler, R., Stemple, D.L., Smith, J.C., and Wilson, S.W. 2000. Silberblick/Wnt11 mediates convergent extension movements during zebrafish gastrulation. Nature 405: 7681.

Hemmati-Brivanlou, A. and Melton, D.A. 1994. Inhibition of activin receptor signaling promotes neuralization in Xeno- 
pus. Cell 77: 273-281.

Hemmati-Brivanlou, A., de la Torre, J.R., Holt, C., and Harland, R.M. 1991. Cephalic expression and molecular characterization of Xenopus En-2. Development 111: 715-724.

Henry, G.L., Brivanlou, I.H., Kessler, D.S., Hemmati-Brivanlou, A., and Melton, D.A. 1996. TGF-beta signals and a pattern in Xenopus laevis endodermal development. Development 122: 1007-1015.

Isaacs, H.V., Pownall, M.E., and Slack, J.M. 1994. eFGF regulates Xbra expression during Xenopus gastrulation. EMBO J. 13: 4469-4481.

Keller, R. 1991. Early embryonic development of Xenopus laevis. Methods Cell Biol. 36: 61-113.

Keller, R., Shih, J., and Domingo, C. 1992. The patterning and functioning of protrusive activity during convergence and extension of the Xenopus organiser. Development (1992 Supplement) : 81-91.

Kim, S.H., Yamamoto, A., Bouwmeester, T., Agius, E., and Robertis, E.M. 1998. The role of paraxial protocadherin in selective adhesion and cell movements of the mesoderm during Xenopus gastrulation. Development 125: 4681-4690.

Kimelman, D. and Griffin, K.J. 2000. Vertebrate mesendoderm induction and patterning. Curr. Opin. Genet. Dev. 10:350356.

Kramer, S., Okabe, M., Hacohen, N., Krasnow, M.A., and Hiromi, Y. 1999. Sprouty: A common antagonist of FGF and EGF signaling pathways in Drosophila. Development 126: 2515-2525.

Kroll, K.L. and Amaya, E. 1996. Transgenic Xenopus embryos from sperm nuclear transplantations reveal FGF signaling requirements during gastrulation. Development 122: 31733183.

Lee, P.L., Johnson, D.E., Cousens, L.S., Fried, V.A., and Williams, L.T. 1989. Purification and complementary DNA cloning of a receptor for basic fibroblast growth factor. Science 245: 57-60.

MacNicol, A.M., Muslin, A.J., and Williams, L.T. 1993. Raf-1 kinase is essential for early Xenopus development and mediates the induction of mesoderm by FGF. Cell 73: 571-583.

Minowada, G., Jarvis, L.A., Chi, C.L., Neubuser, A., Sun, X., Hacohen, N. Krasnow, M.A., and Martin, G.R. 1999. Vertebrate Sprouty genes are induced by FGF signaling and can cause chondrodysplasia when overexpressed. Development 126: 4465-4475.

Mohammadi, M., Dionne, C.A., Li, W., Li, N., Spivak, T., Honegger, A.M., Jaye, M., and Schlessinger, J. 1992. Point mutation in FGF receptor eliminates phosphatidylinositol hydrolysis without affecting mitogenesis. Nature 358: 681684.

Mohammadi, M., Dikic, I., Sorokin, A., Burgess, W.H., Jaye, M., and Schlessinger, J. 1996. Identification of six novel autophosphorylation sites on fibroblast growth factor receptor 1 and elucidation of their importance in receptor activation and signal transduction. Mol. Cell. Biol. 16:977-989.

Musci, T.J., Amaya, E., and Kirschner, M.W. 1990. Regulation of the fibroblast growth factor receptor in early Xenopus embryos. Proc. Natl. Acad. Sci. 87: 8365-8369.

Muslin, A.J., Peters, K.G., and Williams, L.T. 1994. Direct activation of phospholipase C-gamma by fibroblast growth factor receptor is not required for mesoderm induction in Xenopus animal caps. Mol. Cell. Biol. 14: 3006-3012.

Pawson, T. and Saxton, T.M. 1999. Signaling networks-Do all roads lead to the same genes? Cell 97: 675-678.

Peters, K.G., Marie, J. Wilson, E., Ives, H.E., Escobedo, J., Del Rosario, M., Mirda, D., and Williams, L.T. 1992. Point mutation of an FGF receptor abolishes phosphatidylinositol turnover and $\mathrm{Ca} 2+$ flux but not mitogenesis. Nature 358: 678-681.

Pownall, M.E., Tucker, A.S., Slack, J.M., and Isaacs, H.V. 1996. eFGF, Xcad3 and Hox genes form a molecular pathway that establishes the anteroposterior axis in Xenopus. Development 122: 3881-3892.

Pownall, M.E., Isaacs, H.V., and Slack, J.M. 1998. Two phases of Hox gene regulation during early Xenopus development. Curr. Biol. 8: 673-676.

Reich, A., Sapir, A., and Shilo, B. 1999. Sprouty is a general inhibitor of receptor tyrosine kinase signaling. Development 126: 4139-4147.

Rossant, J., Ciruna, B., and Partanen, J. 1997. FGF signaling in mouse gastrulation and anteroposterior patterning. Cold Spring Harbor Symp. Quant. Biol. 62: 127-133.

Ryan, P.J., Paterno, G.D., and Gillespie, L.L. 1998. Identification of phosphorylated proteins associated with the fibroblast growth factor receptor type I during early Xenopus development. Biochem. Biophys. Res. Commun. 244: 763-767.

Schulte-Merker, S. and Smith, J.C. 1995. Mesoderm formation in response to Brachyury requires FGF signalling. Curr. Biol. 5: 62-67.

Shih, J. and Keller, R. 1992. Patterns of cell motility in the organizer and dorsal mesoderm of Xenopus laevis. Development 116: 915-930.

Simon, M.A. 2000. Receptor tyrosine kinases: Specific outcomes from general signals. Cell 103: 13-15.

Slusarski, D.C., Yang-Snyder, J., Busa, W.B., and Moon, R.T. 1997. Modulation of embryonic intracellular Ca2+ signaling by Wnt-5A. Dev. Biol. 182: 114-120.

Smith, J.C., Price, B.M., Green, J.B., Weigel, D., and Herrmann, B.G. 1991. Expression of a Xenopus homolog of Brachyury $(\mathrm{T})$ is an immediate-early response to mesoderm induction. Cell 67: 79-87.

Smith, W.C. and Harland, R.M. 1991. Injected Xwnt-8 RNA acts early in Xenopus embryos to promote formation of a vegetal dorsalizing center. Cell 67: 753-765.

Sokol, S.Y. 1996. Analysis of Dishevelled signalling pathways during Xenopus development. Curr. Biol. 6: 1456-1467.

- 1999. Wnt signaling and dorso-ventral axis specification in vertebrates. Curr. Opin. Genet. Dev. 9: 405-410.

Song, J. and Slack, J.M. 1996. XFGF-9: A new fibroblast growth factor from Xenopus embryos. Dev. Dyn. 206: 427-436.

Sun, X., Meyers, E.N., Lewandoski, M., and Martin, G.R. 1999. Targeted disruption of Fgf8 causes failure of cell migration in the gastrulating mouse embryo. Genes \& Dev. 13:18341846.

Tada, M. and Smith, J.C. 2000. Xwnt11 is a target of Xenopus Brachyury: Regulation of gastrulation movements via Dishevelled, but not through the canonical Wnt pathway. Development 127: 2227-2238.

Tang, T.L., Freeman, Jr., R.M., O'Reilly, A.M., Neel, B.G., and Sokol, S.Y. 1995. The SH2-containing protein-tyrosine phosphatase SH-PTP2 is required upstream of MAP kinase for early Xenopus development. Cell 80: 473-483.

Tefft, J.D., Lee, M., Smith, S., Leinwand, M., Zhao, J., Bringas, Jr., P., Crowe, D.L., and Warburton, D. 1999. Conserved function of mSpry-2, a murine homolog of Drosophila sprouty, which negatively modulates respiratory organogenesis. Curr. Biol. 9: 219-222.

Umbhauer, M., Marshall, C.J., Mason, C.S., Old, R.W., and Smith, J.C. 1995. Mesoderm induction in Xenopus caused by activation of MAP kinase. Nature 376: 58-62.

von Dassow, G., Schmidt, J.E., and Kimelman, D. 1993. Induction of the Xenopus organizer: Expression and regulation of Xnot, a novel FGF and activin-regulated homeo box gene. 
Nutt et al.

Genes \& Dev. 7: 355-366.

Wacker, S., Brodbeck, A., Lemaire, P., Niehrs, C., and Winklbauer, R. 1998. Patterns and control of cell motility in the Xenopus gastrula. Development 125: 1931-1942.

Wallingford, J.B., Rowning, B.A., Vogeli, K.M., Rothbacher, U., Fraser, S.E., and Harland, R.M. 2000. Dishevelled controls cell polarity during Xenopus gastrulation. Nature 405: 8185.

Weinstein, D.C., Marden, J., Carnevali, F., and HemmatiBrivanlou, A. 1998. FGF-mediated mesoderm induction involves the Src-family kinase Laloo. Nature 394: 904-908.

Whitman, M. and Melton, D.A. 1992. Involvement of p21ras in Xenopus mesoderm induction. Nature 357: 252-254.

Yamaguchi, T.P., Harpal, K., Henkemeyer, M., and Rossant, J. 1994. fgfr-1 is required for embryonic growth and mesodermal patterning during mouse gastrulation. Genes \& Dev. 8: $3032-3044$ 


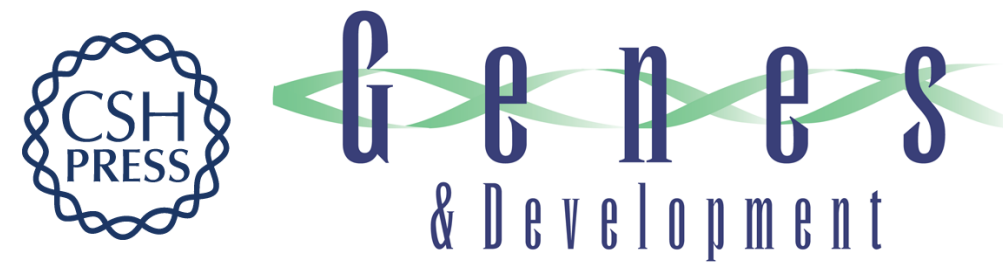

\section{Xenopus Sprouty2 inhibits FGF-mediated gastrulation movements but does not affect mesoderm induction and patterning}

Stephen L. Nutt, Kevin S. Dingwell, Christine E. Holt, et al.

Genes Dev. 2001, 15:

Access the most recent version at doi:10.1101/gad.191301

References This article cites 63 articles, 28 of which can be accessed free at: http://genesdev.cshlp.org/content/15/9/1152.full.html\#ref-list-1

License

Email Alerting

Receive free email alerts when new articles cite this article - sign up in the box at the top Service right corner of the article or click here.

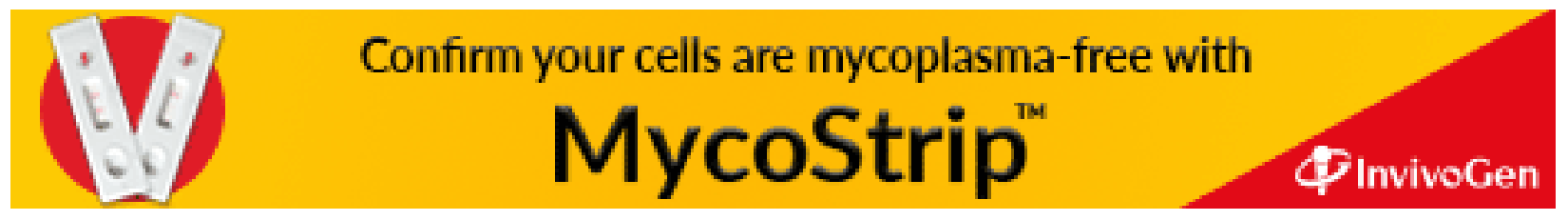

